\title{
Bosquejo histórico de los drenajes en cirugía y sus autores
}

\author{
A brief historical review of drains and their authors \\ Gilberto Guzmán-Valdivia Gómez,* Shantalle Maribell Arellano-Lomelí**
}

Palabras clave: Drenaje, drenaje quirúrgico, historia.

Key words: Drain, surgical drainage, history.
* Jefe de Investigación. ** Médica pasante en servicio social.

Unidad de Investigación. Escuela Mexicana de Medicina de la Universidad La Salle.

Recibido: 05/04/2016 Aceptado: 12/05/2016

\section{RESUMEN}

Los sistemas de drenaje son instrumentos que permiten la salida de colecciones líquidas o gaseosas, naturales o patológicas, que el médico cirujano requiere extraer del organismo; el instrumento se denomina dren. A través de la historia, se han desarrollado diversos sistemas con diferentes drenes en cuanto a su forma, diseño, material y modo de drenar. La necesidad de extracción de estas colecciones más el ingenio de quien los diseñó permitieron la evolución a los sistemas de drenaje actuales. El propósito del presente artículo es presentar cómo fueron apareciendo los drenajes, el material disponible para manufacturar los drenes y nombrar en su contexto a quienes fueron los autores de los mismos.

\section{ABSTRACT}

Drainage systems are instruments which allow the removal of liquid or gas, natural or pathological collections that the physician needs to remove from the body; the instrument is called drain. Throughout history, various systems have been developed with different drains in terms of their form, design, material and draining mode. The need to remove these collections and the ingenuity of those who designed them allowed the evolution of current drainage systems. The purpose of this article is to present how drains appeared, the materials available to manufacture them, and to name who their authors were.
$\mathrm{E}_{\mathrm{q}}^{\prime}$ l concepto drenaje se refiere a la acción que tiene como finalidad la eliminación de líquidos, gases, secreciones o derrames naturales o patológicos de una herida, de un absceso incidido, aunque no completamente vacío, o de una cavidad a través de un instrumento denominado dren. Por lo tanto, no es lo mismo hablar de drenajes y de drenes, aunque en nuestra práctica rutinaria nos refiramos al drenaje como este medio, aditamento o instrumento. ${ }^{1-4}$ Tal vez sea el momento de cambiar el concepto.

También se ha denominado drenaje a las comunicaciones internas realizadas quirúrgicamente entre cavidades y/o epitelios, como lo es el drenaje interno de un pseudoquiste pancreático, la interposición de estructuras anatómicas, como el llamado "drenaje de Voelcker", que en realidad es una derivación biliodigestiva, o la colocación de endoprótesis como en la vía biliar, o drenajes de estructuras específicas como el drenaje de Kehr, que es la sonda en T en la vía biliar.

En este trabajo nos referiremos al drenaje como aquel sistema en el que se utiliza un dren para eliminar líquidos y gases patológicos o naturales que se producen en exceso y cuya extracción se requiere como método terapéutico, así como aquel que el cirujano deja con la intención de incrementar la seguridad de su cirugía, llamado drenaje profiláctico.

Pero, ¿de dónde viene la idea de construir o diseñar un método de drenaje?, ¿quiénes fueron los autores? La respuesta a estas preguntas es el motivo del presente artículo.

Se realizó una revisión sistemática del tema a través de una búsqueda electrónica en las bases de datos de Medline/PubMed y en EBSCOhots; se identificaron los artículos que describían aspectos históricos de los drenajes quirúrgicos. Los siguientes términos fueron utilizados: drain, dren; drainage, drenaje; surgical 
drainage, drenaje quirúrgico; intraperitoneal drainage, drenaje intraperitoneal; abdominal drainage, drenaje abdominal, y prophylactic drainage, drenaje profiláctico. Posteriormente, de ser necesario, se realizaría la investigación bibliográfica de algunos detalles en otros servidores.

El propósito de la búsqueda fue encontrar los aspectos históricos referidos, tratar de establecer el contexto del autor con el diseño de sus drenajes y el material utilizado, así como la intención o indicación para la colocación del dren.

Son muchos los personajes en la historia de la medicina que han utilizado el sistema de drenaje o drenes, por lo que seguramente nos faltará nombrar a alguien.

Se ha referido que el primer reporte de uso del drenaje fue hecho por Hipócrates de Cos (460-377 a. de C.), médico de la antigua Grecia, considerado como padre de la medicina por sus grandes aportaciones a ella, principalmente, su estudio sistemático de la medicina clínica. Describió en los Tratados hipocráticos, colección también conocida como Corpus hippocraticum, la colocación de un drenaje en el tórax para drenar un empiema. No se refiere el material utilizado, pero sí a la técnica de colocación. ${ }^{5-7}$

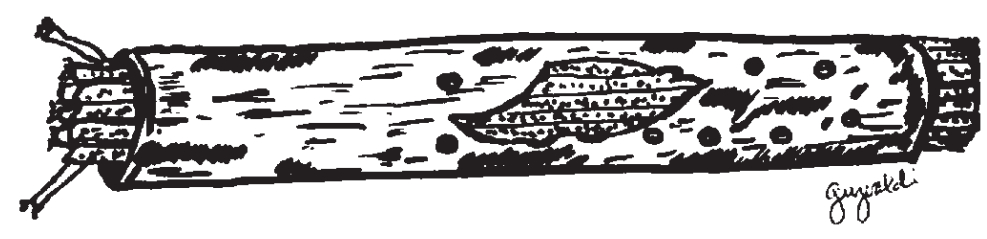

Figura 1. Dren diseñado por Guy Chauliac: cilindro metálico multiperforado con tiras de lino en su interior.

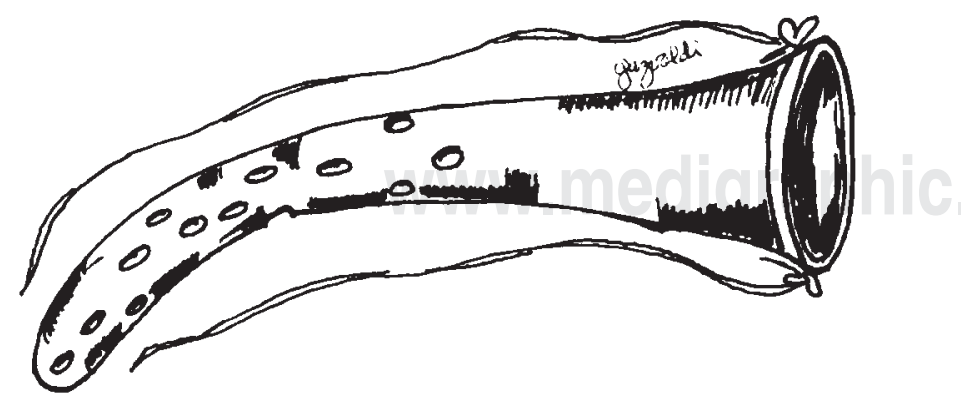

Figura 2. Dren de Ambrosio Paré: tubo de metal cónico y curvo para evitar su deslizamiento y con tiras para su fijación.
Aurelio Celso (25 a. de C.-50 d. de C.), enciclopedista romano que refirió haber estudiado los escritos de Hipócrates, utilizó tubos cónicos de plomo o latón con tapones ajustables para el tratamiento de la ascitis. Refería que los tubos debían ser cónicos para evitar su deslizamiento hacia la cavidad abdominal y los tapones para llevar a cabo el drenaje intermitente del líquido de ascitis. Celso fue autor de la obra enciclopédica De re medica, todo un tratado de las enfermedades y sus tratamientos, que estuvo perdido hasta la Edad Media. ${ }^{8,9}$

Después de estos dos reportes, el uso de drenajes y drenes es reconocido hasta el año de 1363, en el que el cirujano y anatomista francés Guy Chauliac, el más prominente cirujano de la Europa medieval, catalogado por muchos como el padre de la cirugía, describió en su obra Chirurgia Magna los drenes hechos con tiras de lino dentro de un cilindro metálico en forma de mecha para mejorar el drenaje, evitar el cierre de la herida y favorecer la dilatación de su trayecto (Figura 1). Chirurgia Magna fue el texto de referencia por cuatro siglos, el mejor desde los tiempos de Galeno (siglo II d. de C.). ${ }^{7}$

Ambrosio Paré (1510-1590), cirujano francés con mucha actividad médica y quirúrgica en guerras, entre muchas aportaciones incluyó el cuidado de las heridas y la utilización de tubos para drenar abscesos. Los tubos eran tanto de oro y plata como de plomo y latón; eran curvos y con agujeros; dejaba un hilo tutor para evitar la migración del dren a la cavidad abdominal (Figura 2).5,6,10

Johannes Scultetus (en latín) o Johann Schulte (en alemán) nació en la ciudad de Ulm, Alemania, en 1595. En su obra Armamentarium Chirurgicum mostraba un gran catálogo de instrumentos quirúrgicos, procedimientos y reportes de casos. Aquí refiere la mayor eficacia en drenar colocando una mecha de lino en el interior de los tubos de drenaje, como lo hacía Chauliac, pero reconociendo que el drenaje era eficiente no sólo por los tubos, sino por la tela que contenía. Así, se le reconoce como el primero en recomendar el principio de capilaridad, aunque la descripción de este fenómeno la informó el físico británico James Jurin en 1718 con tubos capilares. $7,8,11$

Lorenz Heister (1683-1758), notabilísimo anatomista y cirujano alemán, un siglo después 
siguió impetuosamente este principio y lo explicó detalladamente en su obra Chirurgie, publicada en 1739, por lo que también se le ha considerado el introductor del drenaje por capilaridad, principio precursor del drenaje de Penrose (Figura 3).8,12

En 1809, Ephraim McDowell, cirujano norteamericano y pionero de la cirugía en ese país, exteriorizó una ligadura insertada en un ovario para favorecer el drenaje por capilaridad.

Hasta ese momento, los drenes utilizados eran rígidos y metálicos, rectos o curvos, asegurados con sutura y con mechas de lino para favorecer el drenaje.

El primer cirujano en utilizar un tubo hueco de goma fue el profesor Edmund Randolph Peaslee, norteamericano, miembro de la Sociedad Médica de New Hampshire. En 1855, reportó ante esta sociedad el uso de tubos de caucho para drenar la cavidad pélvica a través de la vagina. Sus resultados no fueron satisfactorios, más por la vía de drenaje que por los drenes utilizados. ${ }^{7,8}$

El francés Marie E Chassaignac, en su Tratado de la supuración y drenaje quirúrgico, publicado en 1859, difundió el uso de tubos de goma o caucho en el drenaje de abscesos, drenes precursores de las sondas de Nélaton actuales. ${ }^{13}$

Eugene Koeberlè de Estrasburgo, Francia, pionero de la cirugía de ovario e histerectomía, así como del uso de las pinzas hemostáticas,

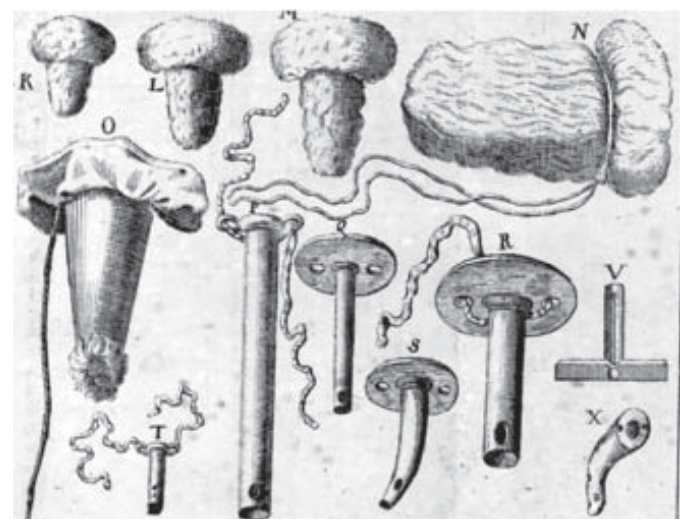

Tomado de: http://tomascabacas.com/tag/apositos-y-vendasgrabado-de-heister-siglo-xviiil

Figura 3. Diferentes tapones, apósitos y drenes de Heister. Diferentes formas y tamaños. en 1867 introdujo los tubos de vidrio, cerrados en su extremo distal, pero con orificios en las paredes laterales. Los resultados no fueron los esperados, ya que estos drenes se tapaban con epiplón o asas de intestino. ${ }^{14}$

Joseph Lister (1827-1912), cirujano inglés y quien desarrolló la cirugía antiséptica, estuvo muy de acuerdo con la colocación de drenes; dijo: "es imposible extremar la importancia de los tubos de drenaje". Recomendó el uso de pelo de caballo alrededor del tubo de drenaje para facilitar su extracción. Lord Lister, posteriormente, comenzó a utilizar tubos de caucho, les hizo agujeros en su trayecto, sustituyó el pelo de caballo por hilos de seda e introducía franjas de lino que remojaba en solución antiséptica de ácido carbólico dentro del tubo. 5,6,15

William Halsted (1852-1922), cirujano norteamericano considerado como uno de los padres de la cirugía moderna, usó goma natural de la planta gutapercha, la cual cubría a una mecha de gasas de algodón. ${ }^{7,8}$

Los materiales utilizados hasta estos años incluyeron madejas de lino, gasa, huesos de buey descalcificados, huesos de ave de corral, caucho o hule, gasa enrollada dentro de una lámina de hule o goma, tubos de vidrio, tubos de metal e, incluso, aorta de buey. Para mejorar el sistema de drenaje dentro de los tubos, se introducían también pelos de caballo, catgut y cuerdas de lana en forma de pabilos. ${ }^{7}$

Jan Mikulicz (1850-1905), que nació en la actual Ucrania (el Imperio Austriaco de aquel entonces), condenó la utilización de tubos y cualquier forma de irrigación a través de ellos. Introdujo un nuevo tipo de dren hecho de una lámina fenestrada de goma llena de tiras largas de gasa impregnadas con tintura de iodo. Las secreciones eran absorbidas por la gasa, pero la recomendación de Lister para el uso de este objeto era para obliterar espacios muertos. En nuestros días, el uso de este taponamiento, ya sea con gasas o compresas, es para empaquetar espacios con fines hemostáticos, como lo demuestra la figura 4 (empaquetamiento tipo Mikulicz). ${ }^{16}$

Auguste Nélaton (1807-1873), cirujano que nació en París, contribuyó a introducir varias innovaciones instrumentales; por ejemplo, la sonda exploratoria, que es un estilete que en uno de sus extremos contiene una bolita de 


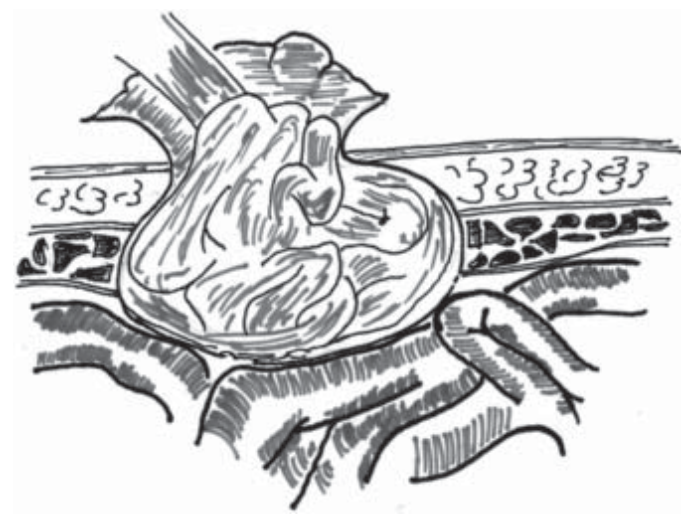

Figura 4. Drenaje de Mikulicz.

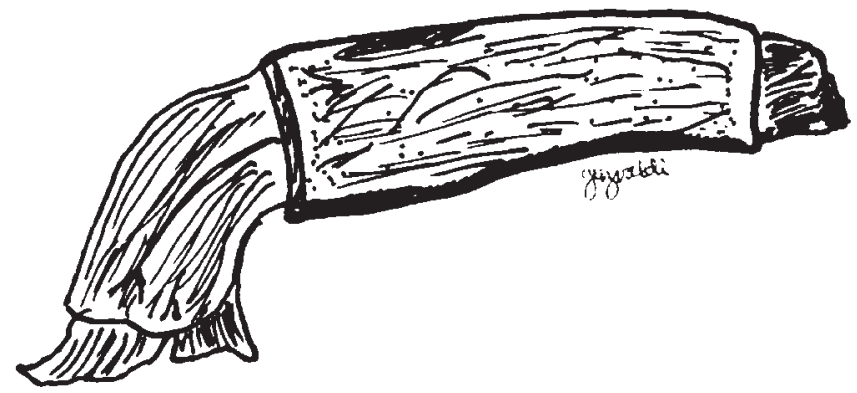

Figura 5. Dren de Penrose: condón de caucho cortado en su punta con gasa de algodón en su interior.

porcelana deslustrada para la investigación de trayectos de proyectiles de plomo, así como la sonda flexible que actualmente lleva su nombre. ${ }^{17}$

En los años 1890, el uso de tubos de drenaje, tanto de vidrio, como de caucho, se hizo común en los Estados Unidos de América; sin embargo, se observó la presencia de complicaciones por su uso, como hernias abdominales, obstrucción intestinal, incremento en la formación de adherencias, fístulas fecales, persistencia de tracto fistuloso e infección. Estos aspectos inspiraron al Dr. Hunter Robb, cirujano del Hospital John Hopkins, a investigar la contaminación de las heridas y la infección a través de los drenes, y reportó en 1891 el estudio bacteriológico de los sistemas de drenaje, recomendando la limpieza constante de los tubos de vidrio y caucho, así como de las ropas que los cubren..$^{8,18}$

Charles Bringham Penrose, profesor de ginecobstetricia de la Universidad de Pennsyl- vania observó la dificultad de la extracción del tubo rígido y en su texto Enfermedades de la mujer describió un nuevo modelo de dren que consistía en un condón - que en aquella época era de caucho vulcanizado- que cortaba en su extremo distal, por lo que quedaba abierto en sus dos extremos; colocaba una gasa de algodón en su interior, lo que en nuestros días es llamado "drenaje en cigarrillo" (Figura 5). Este dren era, por lo tanto, mucho más flexible, permitía el drenaje por capilaridad a través de la gasa y facilitaba su extracción con mucho menos dolor. ${ }^{7,8}$

Este drenaje flexible es el precursor de los drenes de látex que se utilizan comúnmente en el presente; se les denomina Penrose aunque no lleven gasa en su interior.

En 1898, George Heaton, cirujano inglés del Hospital de Birmingham, observó que el drenaje de sangre, pus y orina mojaba la ropa que cubre los drenes; en consecuencia, también las ropas de cama, y les producía escalofríos a los pacientes. Presentó ante la sociedad médica británica la idea de utilizar un sistema de sifón utilizado por los dentistas durante las operaciones en la boca para conservar la ropa seca. El principio del aparato es mantener una acción de sifón leve y continua a través de una bomba modificada de Sprengel (bomba que utilizaban los químicos con principios de vacío) que conectaba a un tubo de vidrio, que se colocaba dentro de otro tubo perforado en forma de bulbo. Este sistema era muy voluminoso e incómodo, pero se trata del precursor de los sistemas de drenaje con aspiración (Figura 6). ${ }^{19}$

En 1905, John Lawrence Yates, de Wisconsin, publicó el resultado de sus experimentos en animales y concluyó que el exudado seroso obtenido por un dren abdominal era causado por la reacción a cuerpo extraño del dren y concluyó que "el drenaje de la cavidad abdominal es física y fisiológicamente imposible" ${ }^{20}$

La experiencia durante la Primera Guerra Mundial (de 1914 a 1918) disminuyó el uso y abuso de los drenajes, sobre todo en Europa.

En la década de 1930 se empezó a popularizar el uso de los sistemas de drenaje por succión. RC Chaffin, cirujano norteamericano dedicado a la ginecobstetricia en California, describió en 1932 uno nuevo, refiriendo que el drenaje por gravedad es insuficiente. El dren se comercializó en 1934, diseñado para drenar abscesos profun- 


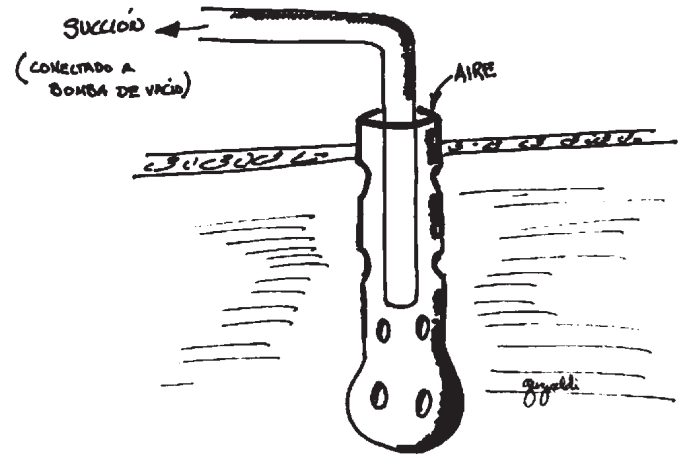

Figura 6. Sistema de drenaje por aspiración de Heaton. Nótese el sistema de doble tubo de vidrio para favorecer la succión.

dos de la cavidad pélvica y fístulas vesicales. Este drenaje consistía en un sistema de un tubo rígido de doble lumen en el que por una luz entra aire y por la otra se aspira, manteniendo presión positiva en el espacio drenado y facilitando la extracción de la colección con una succión lenta e intermitente a través de una jeringa. ${ }^{7,8}$ El sistema de drenaje se hizo popular y Chaffin lo reforzó en su publicación de 1945. ${ }^{21}$

John Benjamin Murphy, ${ }^{6}$ cirujano estadounidense reconocido por varios epónimos (como el punto y signo de Murphy), en 1947 utilizó este sistema de succión para cerrar espacios muertos después de una mastectomía. Al ver la utilidad del drenaje por succión, los doctores Baron —en 1950_y Raffl —en 1952_22 diseñaron paralelamente un sistema de drenaje cerrado y con succión continua empleando una fuente externa de vacío (aparato de Wangensteen) voluminosa que impedía la movilización del paciente.

Mientras se buscaba un dispositivo de succión más práctico, pequeño y donde la cantidad de lo drenado pudiera cuantificarse, se regresó al uso de drenes de látex, suaves y flexibles; el drenaje de Penrose de nuestros días era muy utilizado en Estados Unidos en aquella época de 1950 y 1960, principalmente después de colecistectomías; se recomendaba su retiro antes de las 48 horas por su acción irritativa. ${ }^{7}$

En 1954, el cirujano francés Henri Redon introdujo un tubo de poliuretano multiperforado y - siguiendo la idea de su interno Guy Jost, según manifiesta el mismo Redon—diseñó una unidad de succión que era portátil. Era inicialmente utilizada en cirugías del cerebro y, posteriormente, en mastectomías, vaciamientos ganglionares de axila, ingle y cuello; fue el precursor de los modernos sistemas de drenaje, incluyendo el de aspiración pleural. ${ }^{23,24}$

En 1959 aparece el caucho siliconado, actualmente llamado silastic. En 1961, la compañía Firestone Rubber fabricó el primer dren de látex radiopaco agregándole bario durante su proceso de fabricación. ${ }^{8}$

Los drenajes cerrados con sistema de succión (Redon, Choffin, Baron y Raffl) fueron ganando terreno al observarse su mayor eficacia en la acción de drenar, pero se tapaban con asas de intestino o epiplón o dañaban las anastomosis cuando se les colocaba cerca, aunque se les vio utilidad para cerrar espacios después de grandes disecciones en cirugía de mama, pared torácica y abdominal, así como en colgajos realizados en cualquier parte del cuerpo. ${ }^{25,26}$

Para disminuir los problemas ocasionados por los sistemas de drenaje con succión, se idearon los drenes de doble o triple lumen; los espacios entre ellos sirvieron como canales de introducción de aire a la cavidad, lo que favorecía el sistema de sifón que evitaba el colapso del dren y el taponamiento de los agujeros, daba mayor eficiencia en el drenaje y evitaba la contaminación de la herida quirúrgica. ${ }^{27}$

A principios de 1970, se mejoró el sistema de vacío de Redon. Los doctores Frederick E Jackson, jefe del Departamento de Neurocirugía del Hospital Naval del Campo de Pendleton, en California, y Richard A Pratt III, neurocirujano del mismo hospital, idearon un dren doble de silicón multiperforado con una pequeña cresta interna para darle rigidez y evitar así su colapso; tiene un sistema de succión a través de un bulbo autoexpandible y sus sistemas de tubos permiten la entrada de pequeñas cantidades de aire para equilibrar la presión atmosférica en la cavidad drenada. Se inició su uso en cirugía craneana y, posteriormente, se universalizó en otras cavidades. ${ }^{28}$

En el presente, el drenaje de Jackson-Pratt se ha mejorado con la adición de válvulas unidireccionales que permiten la salida del líquido aspirado y disminuyen la migración de las bacterias al interior del organismo; incluso, se fabrican con tres o más lúmenes, uno de ellos para irrigación. Actualmente, los drenes son de 


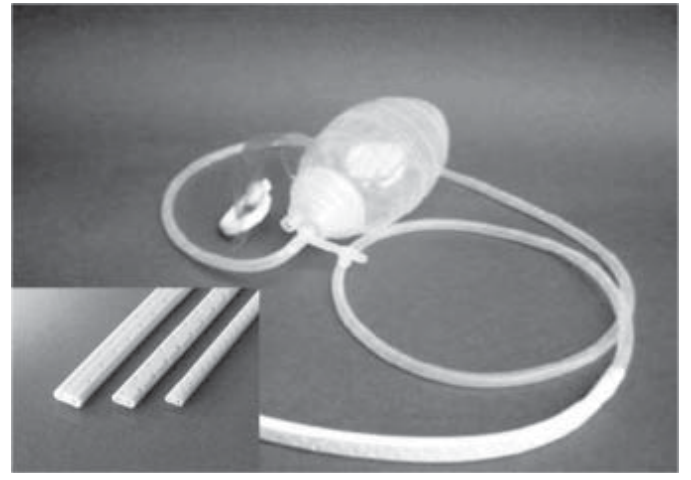

Figura 7. Dren de Jackson-Pratt moderno: tubo de silicón multiperforado, puede ser plano o redondo. Las crestas internas le dan rigidez para evitar su colapso.

poliuretano y silicón o silastic, que son suaves, flexibles, fácilmente manipulables, provocan poca reacción, difícilmente se tapan y permiten la cuantificación exacta de lo drenado (Figura 7). La comercialización de estos drenes se ejemplifica con el Drenovac, Biovac y Hemovac, entre otros, con tubos redondos o planos.

\section{REFERENCIAS}

1. Segatore L. Diccionario Médico. 4. a ed. Barcelona, España: Editorial Teide; 1971. p. 361.

2. Correa MCE. Diccionario de Ciencias Médicas. MéxiCo: IPSO editores; 1981. p. 88.

3. Diccionario Médico Roche. España: Editorial DOYMA; 1993.

4. Diccionario de Medicina de la Facultad de Medicina de la Universidad de Navarra. Madrid España: Editorial Espasa Calpe; 1999. p. 382.

5. Moss JP. Historical and current perspectives on surgical drainage. Surg Gynecol Obstet. 1981; 152: 517-525.

6. Levy M. Intraperitoneal drainage. Am J Surg. 1984; 147: 309-314.

7. Robinson JO. Surgical drainage: an historical perspective. Br J Surg. 1986; 73: 422-426.

8. Memon MA, Memon MI, Donohue JH. Abdominal drains: a brief historical review. Ir Med J. 2001; 94: 164-166.

9. Pérez-González D. Textos clásicos de medicina. Medicina: Historia y Filosofía. [Internet.] [Revisado el 23 de marzo de 2016.] Disponible en: http://hyfmedbuap. blogspot.mx/2008/01/aulo-cornelio-celso

10. Cid F. Breve historia de las ciencias médicas. $3 .^{\mathrm{a}}$ ed. Barcelona, España: ESPAXS, Publicaciones Médicas; 1990.

11. Strohl EL. Johannes Schultetus (1595-1645). Arch Surg. 1963; 87: 396-397.

12. Cabacas T. Apósitos y vendas. Grabado de Heister. Siglo XVIII. [Internet.] [Revisado el 23 de marzo de 2016.] Disponible en: http://tomascabacas.com/tag/ apositos-y-vendas-grabado-de-heister-siglo-xviii
13. Micó-Navarro JA. Catálogo del Fondo Sánchez Quintanar. En: Cuadernos valencianos de historia de la medicina y de la ciencia. Valencia: Instituto de Estudios y Documentación de la Historia sobre la Ciencia. Universitat de Valencia; 1994. p. 78.

14. Hollander LF. Eugene Koeberlè (1878-1915), founder of modern surgery. Am Chir. 2001; 126: 572-581.

15. Grandes biografías. Lister Joseph, padre de la cirugía antiséptica; método antiséptico. [Internet.] [Revisado el 23 de marzo de 2016.] Disponible en: http://www. historiaybiografias.com/sinfama5/.

16. Historia de la medicina. Jan Mikulicz-Radecki (18501905). [Internet.] [Revisado el 23 de marzo de 2016.] Disponible en: http://www.historiadelamedicina.org/ mikulicz.html

17. Historia de la medicina. Nélaton, Auguste (18071873). [Internet.] [Revisado el 23 de marzo de 2016.] Disponible en: http://www.historiadelamedicina.org/ nelaton.html

18. In memoriam: Hunter Robb. [Internet.] [Revisado el 23 de marzo de 2016.] Disponible en: http://historyof-obgyn.com/uploads/3/5/3/8/3538227//robbhunter1863-1940.pdf

19. Heaton G. Note on the Drainage of Large Cavities after Surgical Operations. [Internet.] [Revisado el 23 de marzo de 2016.] Disponible en: http://www.ncbi. nlm.nih.gov/pmc/articles/PMC2410788

20. Zumtobel V, Ernst R, Senkal M. Effects and side effects of abdominal drainage. In: Treutner KH, Schumpelick V. Peritoneal Adhesions. Berlin: Springer Verlag; 1992. p. 219-222.

21. Chaffin RC. Surgical drainage vs. wicks and venting. Med Times. 1945; 73: 40-45.

22. Raffl $A B$. The use of negative pressure under skin flaps after radical mastectomy. Ann Surg. 1952; 136: 1048.

23. Redon H, Jost G, Troques R. Closure under reduced atmosphere. Mem Academ Chir (Paris). 1954; 80: 394-396.

24. Lesser AJ. The place of wound drainage in surgery with description of a new drain. Arch Surg. 1960; 81: 870-876.

25. Manz CW, Tendres CL, Osaka Y. The detrimental effects of drainage on colonic anastomoses. Dis Colon Rectum. 1970; 13: 17-25.

26. Khan S, Rai P, Misra G. Is prophylactic drainage of peritoneal cavity after gut surgery necessary?: A nonrandomized comparative study from a teaching hospital. J Clin Diagn Res. 2015; 9: PC01-3.doi:10.7860/ JCDR/2015/8293.6577.

27. Durai R, Mownah A, Ng PC. Use of drains in surgery: a review. J Perioper Pract. 2009; 19: 180-186.

28. Jackson FE, Pratt RA. Technical report: a silicone rubber suction drain for drainage of subdural hematomas. Surgery. 1971; 70: 578-579.

Correspondencia:

Gilberto Guzmán-Valdivia Gómez

Unidad de Investigación. Escuela Mexicana de Medicina de la Universidad La Salle. Fuentes Núm. 17, Col. Tlalpan, 14000, Del. Tlalpan, Ciudad de México. Tel: +52(55) 5278-9500, ext. 2157 Tel. móvil: 5585324237 E-mail: gilberto.guzmanvaldivia@ulsa.mx 